\title{
Will AI in pigs become more efficient?
}

J. Roca, I. Parrilla, A. Bolarin, E. A. Martinez and Heriberto Rodriguez-Martinez

\section{Linköping University Post Print}

N.B.: When citing this work, cite the original article.

Original Publication:

J. Roca, I. Parrilla, A. Bolarin, E. A. Martinez and Heriberto Rodriguez-Martinez, Will AI in pigs become more efficient?, 2016, Theriogenology, (86), 1, 187-193.

http://dx.doi.org/10.1016/j.theriogenology.2015.11.026

Copyright: Elsevier

http://www.elsevier.com/

Postprint available at: Linköping University Electronic Press

http://urn.kb.se/resolve?urn=urn:nbn:se:liu:diva-130124

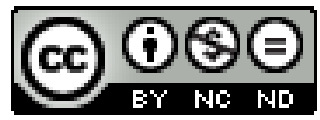




\title{
Will artificial insemination in pigs become more efficient?
}

\section{J Roca ${ }^{1}$, I Parrilla1 ${ }^{1}$ A Bolarin ${ }^{2}$, EA Martinez ${ }^{1}$ and H Rodriguez-Martinez ${ }^{3}$}

${ }^{1}$ Department of Medicine and Animal Surgery, Veterinary Medicine, University of Murcia, Murcia, Spain; ${ }^{2}$ AIM iberica, Topigs Norsvin, Madrid, Spain; ${ }^{3}$ Department of Clinical \& Experimental Medicine (IKE), Linköping University (LiU), Linköping, Sweden.

Corresponding author: Jordi Roca. Tel.: +34868884735; Fax: +34 868887069; E-mail address: roca@um.es

\begin{abstract}
Artificial insemination (AI) is applied worldwide to commercially breed pigs, with fertility outcomes similar to those of natural mating. However, it is not fully efficient, as only liquid-stored semen is used, with a single boar inseminating about 2,000 sows yearly. Use of liquid semen, moreover, constrains international trade and slows genetic improvement. Research efforts, reviewed hereby, are underway to reverse this inefficient scenario. Special attention is paid to studies intended to decrease the number of sperm used per pregnant sow, facilitating the practical use of sexed frozen-thawed semen in swine commercial insemination programs.
\end{abstract}

Keywords: swine, fixed-time artificial insemination, frozen-thawed semen, sexed sperm. 


\section{Introduction}

Production rates and consumption demands for pig meat are continuously increasing worldwide. Production must, therefore, be sustainable and highly efficient while done in a livestock-compatible environment to avoid losing competitiveness [1]. In this context, increasing genetic progress of the swine population to produce more and better meat with fewer bred animals is critical to diminish environmental pressure, both in terms of feedstuff production and manure handling. Reproductive technologies, mainly artificial insemination (AI) and embryo transfer (ET), are the best means to, spreading genetic progress, improve global swine populations [2]. Although considerable progress has recently been made in long-term embryo preservation and non-surgical ET methodologies in pigs, their practical and commercial implementation are yet distant (reviewed in [3]); AI being left as sole practical tool for global pig gene dissemination.

Commercial swine AI started over 50 years ago and it is today extensively used worldwide (reviewed in [4]). During the first 30 years it was more a tool for helping farmers improve breeding management and preventing the spread of venereal diseases rather than a mean to accelerate genetic progress. In this context, effectiveness prevailed over efficiency, which unfortunately still remains -partly- true. The most widely used AIprocedure in swine farms involves use of liquid-stored semen (3-5 days of storage at 15$17^{\circ} \mathrm{C}$ ) and cervical deposition of large sperm numbers per sow/gilt in oestrus (usually 5$7 \times 10^{9}$ sperm in total, as a result of 2-3 AIs per sow/gilt in estrous using 2-3 x10 ${ }^{9}$ sperm per AI-dose) [4]. This AI-procedure is effective, yielding fertility outcomes equal to natural mating. But it is inefficient, limiting the profitability of the most genetically valuable boars, since not more than 2,000 sows/gilts are yearly inseminated per breeding boar. Furthermore, most AI-doses commercially used are derived from semen pools of several (usually 3) boars instead of from a single boar, a procedure that hinders genetic 
progress [5]. This inefficiency contrasts with dairy cattle breeding, where wide use of frozen-thawed (FT) semen makes possible for a single selected breeding bull to inseminate around 50,000 cows yearly [6].

Then, it seems evident that AI-swine should become more focused on widening genetic improvement. We hereby postulate this can be achieved by substantially lowering the number of sperm expended by pregnant sow (which basically increases the number of AI-doses produced/used per single breeding sire) but also by facilitating use of other seminal technologies beyond AI with liquid-stored semen. Those involve use of cryopreserved sperm, with an emphasis on chromosomal-sexed semen. Obviously, focus is on effectiveness, ie maintaining current excellent fertility outcomes. This review is directed to scrutinize how AI-swine is evolving, exploring challenges ahead to become a truly useful and efficient tool to accelerate global genetic progress.

\section{Decreasing sperm numbers used per pregnant sow/gilt}

Drastic decreases in numbers of sperm currently used per achieved pregnancy has seen some progress in recent years. A conventional AI-catheter, which delivers semen AI-dose into the inner folds of cervix, is being gradually replaced by the so-called intrauterine insemination (IUI) catheter, which delivers the AI-dose into the uterine body. The IUI catheter was conceived over 40 years ago but it was not shown useful in commercial swine AI programs until 2000 [7] using 1,500 million sperm per AI-dose. Since then, several reviews discussed in detail particulars of the IUI, including materials, usage, volume and number of sperm per AI-dose and also fertility outcomes (reviewed in [811]). Briefly, the IUI-catheter is cheap, easy to use, does not require special training or extra time per AI, achieving similar as high fertility outcomes as conventional cervical 
AI but using significantly fewer sperm per dose. On average, IUI requires around half the number of sperm per AI-dose than conventional cervical AI when used under similar breeding management conditions, basically because depositing sperm into the uterus body reduces the loss of sperm by backflow [12]. Due to its high fertility, the commercial use of IUI is increasingly widespread in countries with intensive pig breeding and production [11]. Alongside this expansion, many IUI-trials were carried out to define minimum sperm numbers yielding high fertility (based on conventional AI yields). Despite many efforts, there is no yet a standardized minimum sperm number [10]. Looking over the results of IUI-trials it seems obvious that high fertility can be achieved using only 750 million spermatozoa when both farm breeding management and sperm quality of AI-dose are optimal [13]. However, from the same compelling IUI-trials, it also seems clear that the threshold sperm number per IUI-dose that systematically achieves high fertility should be at least $1 \times 10^{9}$ sperm/dose. Further decrease in sperm numbers/AIdose remain challenging since it may compromise fertility outcomes under commercial conditions.

In addition to lowering sperm numbers/AI-dose, efforts are directed towards reducing the number of AIs performed per oestrus. Rozeboom et al. [14] demonstrated that a properly ovulation-timed AI is more important that the number of sperm/AI-dose used. Currently, sows/gilts are inseminated with liquid-stored semen 2 to 3 times per oestrus, averaging between 1.7 and 2.6 AIs depending on the country. Only one of these AIs is effective; the one performed closely to ovulation, in a narrow time window, estimated between $12 \mathrm{~h}$ before and up to $4 \mathrm{~h}$ after ovulation [15]. This single AI would be enough to ensure successful pregnancy, resulting in a significant economic profit as labour time and semen spending decreases. Moreover, the fewer sperm used, the higher the number of AI-doses available, which automatically increases the chances of spreading ejaculates from boars 
with high genetic merit. In addition, a single AI per oestrus would be essential to incorporate use of cryopreserved and sex-sorted semen.

It is thus evident that forecasting the moment of ovulation is critical, which is feasible with a good breeding management, staged in a correct detection and control of oestrus length as it is well know that ovulation occurs about two thirds of oestrus time [16]. An excessive number of AI per oestrus often hides poor breeding management. The accurate detection of oestrus duration is a simple, inexpensive and easy to implement procedure but scarcely used in most swine farms because it is time-consuming as oestrus length varies significantly among sows within the same farm. Oestrus length in weaned sows varies according to the weaning-to-onset of oestrus interval (WOI), being shorter at longer WOIs, and also among farms [17], demanding differential setting of AI-strategies for each WOI and farm. However, this breeding management strategy should not be discarded as its implementation is not risky considering the considerable economic benefits it offers. Most obvious is the elimination of a third insemination without deterioration of fertility outcomes.

Fixed-time AI in sows/gilts with hormonally induced ovulation is the alternative to effectively decrease AI-numbers per oestrus, as it has been praxis for dairy cattle. The procedure is practical because it does not require oestrus detection, decreasing labour costs and increasing reproductive throughput. Noteworthy, a single fixed-time AI per oestrus, which should be the ultimate goal, could be sufficient for a successful pregnancy if the hormonal treatment is well conducted. Many commercially available hormones are available to synchronize ovulation after induction of follicular growth with FSH or their natural agonist eCG, The most effective ones are porcine LH or their natural agonist hCG (both acting at the ovary level) and natural GnRH or their synthetic analogues, such as Buserelin ${ }^{\circledR}$ and Goserelin ${ }^{\circledR}$ (acting at pituitary gland level). For more details, including 
dosage and timetable, see reviews of [18] and [19]. A protocol including porcine LH and single fixed-time AI, $36 \mathrm{~h}$ after porcine LH application resulted in farrowing rates of 86.1 \% and 10.6 piglets born per litter, comparable to control untreated sows and double AI [20]. Similar promising fertility outcomes were achieved recently by Driancourt et al. [21] in both sows and gilts treated with Buserelin ${ }^{\circledR}$ and single fixed-time inseminated at 30-33 h later. Please be aware that some of the above hormones are not commercially available in some countries owing to national regulations. In addition, and for animal welfare reasons, the injectable way for hormonal application is currently under discussion in many state members of the European Union, so alternative application pathways should be explored. Recently, a gel product containing a GnRH agonist (Triptorelin ${ }^{\circledR}$ ) for intravaginal application was evaluated, showing satisfactory results in preliminary trials [22].

A single AI with encapsulated sperm could be another alternative to consider, because it can be performed irrespective of ovulation time. Sperm encapsulation was a sperm technology developed in the 1980s [23] yet currently not commercially applied in livestock. Sperm encapsulation consists in enclosing undiluted semen drops within a semipermeable barium-alginate membrane that allows the exchange of nutrients and metabolites with the medium and that disintegrates slowly, releasing viable sperm gradually over time [24]. Once into the uterus, the slow release of viable sperm ensures an adequate number of functional cells ready to fertilize when ovulation occurs. Swine AI-trials conducted to date are promising, as showed by Vigo et al [25] in a large AI-trial (4,245 AI-sows) achieving farrowing rates of $84.97 \%$ and an average of 12.56 piglets born per litter after a single AI with just $2.5 \times 10^{9}$ encapsulated sperm at the beginning of oestrus. Fertility outcomes are similar to those achieved after 2-3 conventional AIs (82.36 \% of farrowing rate and 12.79 piglets born). However, efforts ought to be made to solve 
some technical questions, linked to preparation, storage and transport of encapsulated sperm, before this sperm technology can be practically used in swine commercial AI programs [24, 26].

\section{Early identification of potentially sub-fertile boars}

Decreasing total sperm numbers per pregnancy pursues the widespread diffusion of genetically high-ranking boars across a greater number of sows/gilts through AI. Certainly, AI-centres contemplate a scenario for the coming years where about 6,000 sows/gilts per year inseminated with ejaculates from just one boar (Data from Artificial Insemination Management Iberica, Spain). To achieve it, changes in the AI-doses production system are necessary, as switching from pooled doses to single-boar doses in order to maximize genetic diffusion of merit boars and, thereby, optimizing breeding farm productivity [5]. Consequently, the current picture of AI-centers is moving from classical small centers housing a few boars usually integrated within breeding farms, towards large, independent and technically highly specialized centers housing a large number of healthy and genetically superior boars that produce, following strict regulations and guidelines to prevent disease spreading, large daily numbers of ready-to-use high-quality liquid-stored AI-doses [27]. This substantial structural progress has also led AI-centers towards more accurate and precise semen assessment by incorporating technologies such as CASA and/or flow cytometry [11], resulting in better fertility outcomes on-farm of the dispensed AI-doses. Despite progress, it is estimated that between 5-7\% subfertile boars still remain "hidden" in AI-centers [27], percentage that could be increased substantially as sperm numbers/AI-dose decreases. It is well known that lowering sperm numbers/AI-dose emerge subfertility problems in boars with previous high fertility using conventional AI- 
doses [28]. Boars and/or ejaculates for AI are normally selected in AI-centres via a standardized spermiogram including sperm numbers, motility and morphology, which is powerful to identify boars/ejaculates with substantial fertility deficiencies but unfortunately unable to identify sub-fertile boars/ejaculates. With this background, AIcentres should supplement current spermiograms with new evaluation assessments able to early identify sub-fertile boars, ideally before incorporated into commercial AIprograms. In addition, such new evaluation assessment should be cost-effective for the AI-centres. Recently, Roca et al. [27] proposed a battery of assessments including sperm nuclear DNA evaluation, cytogenetic analysis for reciprocal translocations and an in vitro fertilization assay that would meet the above requirements as a single assessment for young healthy boars entering AI-centres. Such evaluation could be enough to obtain meaningful estimates of their future in vivo fertility potential.

\section{Daily use of frozen-thawed semen and of sex-sorted sperm in commercial AI- programs}

It is striking, in the XXI century, that practical application of frozen-thawed (FT) semen in commercial swine AI-programs remains a pending target. The swine industry has systematically disregarded its potential benefits against liquid-stored semen, despite its advantages for biosecurity and international exchange in addition to its higher flexibility for use. The clearest evidence of this indifference is that currently only about $1 \%$ of the worldwide total inseminations are performed with FT-semen [29].

The improvements made in cryopreservation methods for the past 15 years, including in composition of freezing and thawing extenders, and the knowledge that not all boars are "good sperm freezers” have shown that AI-doses cryopreserved today show good sperm 
quality and functionality at thawing [30]. Certainly, post-thaw sperm recovery rates over $70 \%$ in motility and viability are now usual, resulting in fertility outcomes near to those achieved with liquid-stored semen. Indeed, Didion et al. [31] reported farrowing rate of $78.7 \%$ and average litter size of 12.5 piglets in a large field AI-trial with more than 2,600 sows intra-cervically inseminated three times per oestrus with AI-doses of $2 \times 10^{9}$ motile sperm ( $4 \times 10^{9}$ total sperm per AI-dose). Consequently, other causes beyond fertility outcomes should explain the currently limited use of FT-semen in swine AI-programs. Almost certainly, the requirements for a larger number of sperm per AI-dose and total sperm expended by pregnant sow together with the complex handling of the AI-dose and the greater variability in fertility outcome, are main causes [4, 9]. Looking at the last cause, Bolarin et al. [32] showed that the inter-AI trials variability in fertility outcomes is higher when using FT-semen compared to liquid-stored semen.

Some tools are already available to overcome the above drawbacks. Deep intrauterine insemination (DUI), delivering the AI-dose in the depth of a uterine horn [33], is a commercially available AI-procedure (DeepBlue AI Catheter ${ }^{\circledR}$, Minitüb, Germany) proved efficient for FT-semen. Although training requirements and device costs prevent their use for liquid-stored semen, the DUI would be the AI-procedure of choice for FTsemen because use of only $1 \times 10^{9}$ total FT-sperm yield fertility outcomes similar to conventional AI-procedure (using $6 \times 10^{9}$ total FT-sperm) [34]. Frozen-thawed spermatozoa have short lifespan (estimated to 6-8 h) once delivered in the female reproductive tract [15]. Thus to reach high fertility AI must be performed within a very brief time window before expected ovulation time. Certainly, Bolarin et al. [35] got farrowing rates of $81.1 \%$ and 9,93 piglets born per litter in sows with hormonally induced ovulation and twice DUI-inseminating with just $1 \times 10^{9}$ sperm per AI-dose close to ovulation time. Interestingly, the same authors reached similar high fertility outcome 
regardless of the total number of FT-sperm per AI-dose ( 1 or $2 \times 10^{9}$ total sperm), proven that inseminating just prior ovulation time is more relevant for the fertility of FT-sperm than the number of sperm delivered/AI-dose. Additionally, the AI-trials carried out by Bolarin et al. [32, 35] also showed that variability in fertility outcomes of FT-sperm among farms, AI-trials and even the season of the year are practically negligible when sows are inseminated shortly before ovulation.

Finally, the complexity in handling frozen AI-doses versus "ready to use" liquid-stored AI-doses, including the obvious careful thawing and also the mandatory subsequent dilution in an appropriate extender to both mitigate cryoprotectant toxicity and fulfil the customary volume of AI-dose, requires training and overtime labour, frustrating the practical usage of FT-semen on-farm. Further research should focus on simplifying procedures. Unfortunately, manufacturers of AI material do not seem too interested to do this, arguing the limited use of FT-semen in the swine AI-programs, which is in itself an incongruity. An example of this lack of interest is the still pending marketing of the FlatPack ${ }^{\circledR}$ packaging system, proven a most suitable cryogenic packaging for boar semen AI-doses [36, 37].

The practical use of sex-sorted spermatozoa in AI-programs would open a new dimension to the swine industry (for more details see 38 and 39). Sexed sperm has itself the potential to substantially expand genetic progress when implemented in commercial AI programs. It allows producing only females thus waiving male-castration, a major animal welfare issue in many countries, particularly in Europe. Currently, the only commercially viable method for sexing sperm is the "Beltsville Sperm Sexing Technology" which involves separation of $\mathrm{X}$ - and $\mathrm{Y}$-chromosome bearing sperm populations by flow cytometry of semen samples previously incubated with the Hoechst $33342(\mathrm{H}-42)$ fluorochrome, that binds to the sperm DNA [40]. The separation of the two sperm populations is possible 
due to the differences in DNA content between $\mathrm{X}$ - and $\mathrm{Y}$-chromosome, where pig $\mathrm{X}$ sperm has a 3.6\% more DNA than the Y-sperm [41]. This small difference in DNA content however hinders the ability of flow cytometers to correctly identify all spermatozoa, making the separation speed slower than expected. Certainly, even in the best current scenario, using high-speed flow cytometers, the number of $\mathrm{X}$ or $\mathrm{Y}$ sperm separated with a purity above 90\% hardly exceeds 20 million per hour [42]. This separation speed is acceptable to produce bovine AI-dose, usually of 2 millions per AIdose when sex-sorted sperm are used [43], but not to produce porcine AI-doses, that must contain at least 500 million sperm. Consequently, enhancing the efficiency of the current procedure is a challenge for the practical use of sex-sorted sperm in commercial swine AI programs. Alternatively, other more efficient procedures than flow cytometry are to be developed. With regard to the first target, research is currently done for identification of Y chromosome-specific sequences by bi-functionalised gold nanoparticles and triplex hybridisation in vivo as well as developing a new laser-controlled deflection system that replaces the deflection of spermatozoa in the electrostatic field [44]. In addition, parallel sorting based on microfluidic chips is being tested to avoid the complex orientation of spermatozoa by the flow-tip, therefore providing much higher rates of sorting [42].

A further obstacle for the commercial application of sex-sorted semen in commercial AIprograms is its special sensitivity to sustain cryopreservation post-sorting. Sex-sorted boar sperm tolerate liquid storage at $15-17^{\circ} \mathrm{C}$ for at least $120 \mathrm{~h}$ [45]. This storage time is, however, insufficient in practice as current flow cytometry procedure requires too long working time to sort sufficient sperm numbers for swine AI-doses, and an additional storage time to send the AI-doses from the sex-sorting lab to farms. Therefore, under this scenario, cryopreservation seems the only useful method to store boar sex-sorted sperm AI-doses. We have demonstrated that sex-sorted and cryopreserved boar spermatozoa 
show acceptable recovery rates in terms of motility and viability after thawing [46], but unfortunately they achieve poor fertility after AI [47]. Consequently, further research, aimed at optimizing cryopreservation protocols is required before sex-sorted sperm can be commercially used. Other compelling constraints for the practical application of sexsorted sperm in commercial swine AI-programs is the ineffectiveness of current AIprocedures. Neither the conventional, requiring at least $1.5 \times 10^{9}$ sperm per AI-dose, or IUI, requiring at least 500 million per AI-dose, are useful. This is due to the fact that, even when employing high-speed sorting flow cytometers, just between 300 and 400 million of sex-sorted spermatozoa can be available each $24 \mathrm{~h}$ per cytometer. Accordingly, alternative AI-procedures requiring much fewer sperm per AI-dose ought to be used. DUI AI-procedure has been postulated as useful onsidering farrowing rates of $47 \%$ with an average of 9.2 piglets born per litter were reached using just 140 million sex-sorted sperm per AI-dose [48]. However, the nowadays still large number of sperm used per AI-dose together with the relatively low fertility limits the possibility of DUI as an efficient AIprocedure for on-farm use of boar sex-sorted sperm. Nevertheless, DUI will be attractive in the future when sorting rates and extend lifespan of sorted sperm are reached.

Nowadays, laparoscopy insemination, despite its high cost and skill requirements, is the only procedure allowing the efficient use of sexed sperm [48]. The procedure involves direct sperm deposition into the utero-tubal junction andor into the tubal ampulla whereby fewer sperm are required for successful fertilization [8]. Recent AI-trials showed farrowing rates of $80 \%$ and average litter sizes of 10.8 piglets born after a single fixedtime laparoscopic insemination in hormonally treated sows placing 1 and 2 million liquidstored sex-sorted sperm in each oviduct and uterine horn, respectively [49]. These excellent fertility results that open for the use of sex-sorted sperm in swine industry, at least at nucleus and multiplier farms where the investment (surgery equipment and trained 
personnel) can be cost-effective, should not prevent further research in developing alternative AI-procedures that allow practical and less expensive ways to use sex-sorted sperm in commercial programs.

\section{Seminal plasma, a mine to fully discover}

Boar ejaculates contain a large amount usually above $150 \mathrm{~mL}$ of seminal plasma (SP). Most SP is discarded during manual collection of the distinct ejaculate fractions or diluted when processing the semen. This handling is now switching towards a growing interest to scrutinize SP-composition and evaluate its relevance for sperm functionality, even fertility. Recent findings support the complex composition of boar SP [50-52] and also demonstrate the relevance of some SP-components, such as Glutathione Peroxidase 5 [53] or Paraoxonase-I [54], for promoting sperm fertility of liquid stored semen IA-doses. In contrast, other SP-components, such as the major proteins PSP-1 and AQN-3, could impair fertility of boar sperm $[53,55]$. This functional contrast between SP-components should be considered before using raw SP as sperm extender of post-thaw semen, action leading to inconsistent fertility results [56, 57].

The relevance of SP on AI-fertility outcomes could be more decisive as AI-centres are moving, for hygienic and labour costs reasons, the traditional procedure of manual ejaculate collection (gloved hand method, which allows to collect separately the sperm rich ejaculate fraction (SRF) poor in SP), to semi-automatic methods, where the entire ejaculate is collected, including the sperm-poorer post-SRF fraction, a fraction containing a larger amount of specially protein-rich SP. The boar SP is especially rich in cytokines (a total of 14 have been recently found in measurable amounts, [52]). Cytokines act as signals for the female immune system once into the female genital tract, modulating the 
maternal tolerance towards embryo and placental development, thus conditioning embryo/foetal development [58]. Exosomes, small extracellular membrane vesicles modulating cellular functionality, also deserve future attention, as they are substantially present in SP. It is speculated that exosomes secreted by the male reproductive tract play important roles in promoting and regulating sperm motility, protecting the sperm in the female genital tract and facilitating fertilizing ability [59].

Together, these findings strongly reveal that improving our knowledge of boar SPcomposition together with an appropriate identification of SP-components involved in sperm functionality and fertility would help predicting the potential fertile of AI-boars and would also be an essential requirement for generating new semen extenders able not only to maintain long time sperm functionality but also and specially to improve sperm fertilizing ability. New extenders for extending lifespan of FT-sperm and for cryopreserving sexed sperm are imperative [30, 47].

\section{Conclusions and future challenges}

Swine AI is clearly progressing but it has still exciting challenges ahead that should substantially help swine industry to achieve desired more efficient production. All challenges point to the same target: to achieve the highest fertility with a single fixedtimed AI using a very small sperm number from the genetically best merited breeding boars, ideally using sexed, cryopreserved sperm. At present, many different research lines are underway to reach this goal, the most notable being those focused in developing new insemination devices, efficient production of pathogens-free and fertile AI-doses, establishing healthy hormonal treatments for ovulation time and improving the efficiency of sperm sexing. However, the swine industry should contribute more decisively for AI 
to become the desired efficient tool, by incorporating the above emergent reproductive technologies, even assuming that its implementation can require some substantial changes in current breeding management programs. Finally, stronger research efforts should focus on the seminal plasma, which seems to contain both the cause and the solution to fertility issues of AI-doses, and which may also open for new perspectives to the successful use of frozen-thawed semen and sexed sperm.

\section{References}

[1] FAO. World agriculture: towards 2015/2030. A fao perspective. Edited by Jelle Bruinsma. 2003. ISBN: 925104835 5. ftp://ftp.fao.org/docrep/fao/005/y4252e/y4252e.pdf

[2] Gerrits RJ, Lunney JK, Johnson LA, Pursel VG, Kraeling RR, Rohrer GA, et al. Perspectives for artificial insemination and genomics to improve global swine populations. Theriogenology 2005;63:283-299.

[3] Martinez EA, Cuello C, Parrilla I, Martinez CA, Nohalez A, Vazquez JL, et al. Recent advances toward the practical application of embryo transfer in pigs Theriogenology. 2015 doi: 10.1016/j.theriogenology 2015.06.002.

[4] Knox RV. Artificial insemination in pigs today. Theriogenology. 2015a Jul 16. doi: 10.1016/j.theriogenology 2015.07.009.

[5] Foxcroft GR, Patterson J, Cameron A, Dyck MK. Application of advanced AI technologies to improve the competi- tiveness of the pork industry. 2010. Proceedings of the 21st IPVS Congress, Vancouver, Canada, July 18-21:25-29.

[6] Vishwanath R. Artificial insemination: the state of the art. Theriogenology 2003;59:571-84.

[7] Watson PF, Behan JR. Intrauterine insemination of sows with reduced sperm numbers: results of a commercially based field trial. Theriogenology 2002,57:1683-93.

[8] Roca J, Hernandez M, Carvajal G, Vazquez JM, Martinez EA. Factors influencing boar sperm cryosurvival. J Anim Sci 2006;84:2692-99. 
[9] Roca J, Parrilla I, Rodriguez-Martinez H, Gil MA, Cuello C, Vazquez JM, et al., Approaches towards efficient use of boar semen in the pig industry. Reprod Domest Anim 2011;46:79-83.

[10] Bortolozzo FP, Menegat MB, Mellagi AP, Bernardi ML, Wentz I. New Artificial Insemination Technologies for Swine. Reprod Domest Anim 2015;50:80-4.

[11] Broekhuijse MLWJ, AH Gaustad AH, Bolarin Guillen, Knol EF. Efficient Boar Semen Production and Genetic Contribution: The Impact of Low-Dose Artificial Insemination on Fertility. Reprod Domest Anim 2015;50:103-9.

[12] Hernández-Caravaca I, Izquierdo-Rico MJ, Matás C, Carvajal JA, Vieira L, Abril D, et al. Reproductive performance and backflow study in cervical and post-cervical artificial insemination in sows. Anim Reprod Sci 2012;136:14-22.

[13] Olesen AK, Hansen C. Intrauterine insemination of sows by using a two-chamber semen bag system. Soc Reprod Fertil 2009;66:81-2.

[14] Rozeboom KJ, Reicks DL, Wilson ME. The reproductive performance and factors affecting onfarm application of low-dose intrauterine deposit of semen in sows. J Anim Sci 2004;82:21648.

[15] Waberski D, Weitze KF, Gleumes T, Schwarz M, Willmen T, Petzoldt R. Effect of time of insemination relative to ovulation on fertility with liquid and frozen boar semen. Theriogenology 1994;42:831-40.

[16] Soede NM, Kemp B. Expression of oestrus and timing of ovulation in pigs. J Reprod Fertil Suppl 1997;52:91-103.

[17] Kemp B, Soede NM. Relationship of weaning-to-estrus interval to timing of ovulation and fertilization in sows. J Anim Sci 1996;74:944-9.

[18] Brüssow KP, Schneider F, Kanitz W, Rátky J, Kauffold J, Wähner M. Studies on fixed-time ovulation induction in the pig. Soc Reprod Fertil Suppl 2009; 66: 187-95.

[19] Kirkwood RN, Kauffold J. Advances in Breeding Management and Use of Ovulation Induction for Fixed-time AI. Reprod Domest Anim 2015;50:85-9. 
[20] Cassar G, Kirkwood RN, Poljak Z; Bennett-Steward K; Robert M. Effect of single or double insemination on fertility of sows bred at an induced estrus and ovulation. J Swine Health 2005;254-8.

[21] Driancourt MA, Cox P, Rubion S, Harnois-Milon G, Kemp B, Soede NM. Induction of an LH surge and ovulation by buserelin (as Receptal) allows breeding of weaned sows with a single fixed-time insemination. Theriogenology 2013;80:391-9.

[22] Knox RV, Taibl JN, Breen SM, Swanson ME, Webel SK. Effects of altering the dose and timing of triptorelin when given as an intravaginal gel for advancing and synchronizing ovulation in weaned sows. Theriogenology 2014;82:379-86.

[23] Nebel RL, Bame JH, Saacke RG, Lim F. Microencapsulation of bovine spermatozoa. Anim Sci 1985;60:1631-9.

[24] Faustini M, Vigo D, Spinaci M, Galeati G, Torre ML. Enhancing insemination performance in pigs through controlled release of encapsulated spermatozoa. Reprod Domest Anim 2012;47:353-8.

[25] Vigo D, Faustini M, Villani S, Orsini F, Bucco M, Chlapanidas T, et al. Semen controlledrelease capsules allow a single artificial insemination in sows. Theriogenology 2009;72:43944.

[26] Perteghella S, Vigani B, Crivelli B, Spinaci M, Galeati G, Bucci D, et al. Sperm Encapsulation from 1985 to Date: Technology Evolution and New Challenges in Swine Reproduction. Reprod Domest Anim 2015;50:98-102.

[27] Roca J, Broekhuijse MLWJ, Parrilla I, Rodriguez-Martinez H, Martinez EA and Bolarin A. Boar Differences In Artificial Insemination Outcomes: Can They Be Minimized? Reprod Domest Anim 2015;50:48-55.

[28] Flowers WL. Increasing fertilization rate of boars: influence of number and quality of spermatozoa inseminated. J Anim Sci 2002;80:E47-53.

[29] Knox RV. The Fertility of Frozen Boar Sperm When used for Artificial Insemination. Reprod Domest Anim 2015b;50:90-97. 
[30] Yeste M. Recent Advances in Boar Sperm Cryopreservation: State of the Art and Current Perspectives. Reprod Domest Anim 2015;50:71-9.

[31] Didion BA, Braun GD, Duggan MV. Field fertility of frozen boar semen: a retrospective report comprising over 2600 AI services spanning a four year period. Anim Reprod Sci. 2013;137:189-96.

[32] Bolarín A, Hernández M, Vazquez JM, Rodriguez-Martinez H, Martinez EA, Roca J. Use of frozen-thawed semen aggravates the summer-autumn infertility of artificially inseminated weaned sows in the Mediterranean region. J Anim Sci 2009;87:3967-75.

[33] Martinez EA, Vazquez JM, Roca J, Lucas X, Gil MA, Parrilla I, Vazquez JL, Day BN. Successful non-surgical deep intrauterine insemination with small numbers of spermatozoa in sows. Reproduction 2001;122:289-96.

[34] Roca J, Carvajal G, Lucas X, Vazquez JM, Martinez EA. Fertility of weaned sows after deep intrauterine insemination with a reduced number of frozen-thawed spermatozoa.Theriogenology 2003;60:77-87.

[35] Bolarín A, Roca J, Rodríguez-Martínez H, Hernández M, Vázquez JM, et al. Dissimilarities in sows' ovarian status at the insemination time could explain differences in fertility between farms when frozen-thawed semen is used. Theriogenology 2006;65:669-80.

[36] Ekwall H, Hernández M, Saravia F, Rodríguez-Martínez H. Cryo-scanning electron microscopy (Cryo-SEM) of boar semen frozen in medium-straws and MiniFlatPacks. Theriogenology 2007;67:1463-72.

[37] Eriksson BM, Petersson H, Rodriguez-Martinez H. Field fertility with exported boar semen frozen in the new flatpack container. Theriogenology 2002;58:1065-79.

[38] Rath D, Johnson LA. Application and commercialization of flow cytometrically sex-sorted semen. Reprod Domest Anim 2008;43:338-46.

[39] Sharpe JC, Evans KM. Advances in flow cytometry for sperm sexing. Theriogenology. 2009;71:4-10.

[40] Johnson LA, Rath D, Vazquez JM, Maxwell WMC, Dobrinsky JR. Pre-selection of sex in swine for production: current status of the process and application. Theriogenology 2005;63:615-24. 
[41] Garner DL. Flow cytometric sexing of mammalian sperm. Theriogenology 2006;65:943-57.

[42] Rath D, Tiedemann D, Gamrad L, Johnson LA, Klein S, Kues W,et al. Sex-Sorted Boar Sperm - An Update on Related Production Methods. Reprod Domest Anim 2015;50:56-60.

[43] Seidel GE Jr. Update on sexed semen technology in cattle. Animal. 2014;8:160-4.

[44] Rath D, Barcikowski S, de Graaf S, Garrels W, Grossfeld R, Klein S, et al. Sex selection of sperm in farm animals: status report and developmental prospects. Reproduction 2013;145:R15-R30.

[45] Alkmin DV, Parrilla I, Tarantini T, Del Olmo D, Vazquez JM, Martinez EA, et al. Seminal plasma affects sperm sex sorting in boars. Reprod Fertil Dev 2014; 28. doi: 10.1071/RD14088.

[46] Parrilla I, del Olmo D, Caballero I, Tarantini T, Cuello C, Gil MA, et al. The effect of glycerol concentrations on the post-thaw in vitro characteristics of cryopreserved sex-sorted boar spermatozoa. Reprod Domest Anim. 2012;47:965-74.

[47] Spinaci M, Perteghella S, Chlapanidas T, Galeati G, Vigo D, Tamanini C, et al., Storage of sexed boar spermatozoa: Limits and perspectives. Theriogenology 2015; 29. doi:10.1016/j.theriogenology.2015.05.018.

[48] Vazquez JM, Parrilla I, Roca J, Gil MA, Cuello C, Vazquez JL, et al. Sex-sorting sperm by flow cytometry in pigs: issues and perspectives. Theriogenology 2009;71:80-8.

[49] Del Olmo D, Parrilla I, Sanchez-Osorio J, Gomis J, Angel MA, Tarantini T, et al. Successful laparoscopic insemination with a very low number of flow cytometrically sorted boar sperm in field conditions. Theriogenology 2014;81:315-320.

[50] Strzeżek J, Wysocki P, Kordan W, Kuklińska M, Mogielnicka M, Soliwoda D, et al., Proteomics of boar seminal plasma - current studies and possibility of their application in biotechnology of animal reproduction. Reprod Biol. 2005;5:279-90.

[51] López Rodríguez A, Rijsselaere T, Beek J, Vyt P, Van Soom A, Maes D. Boar seminal plasma components and their relation with semen quality. Syst Biol Reprod Med 2013;59:5-12.

[52] Barranco I, Ruber M, Perez-Patiño C, Atikuzzaman M, Martínez EA, Roca J, et al. The seminal plasma of the boar is rich in cytokines, with significant individual and Intra-Ejaculate Variation. Am J Reprod Immunol 2015 doi:10.1111/aji.12432. 
[53] Novak S, Ruiz-Sánchez A, Dixon WT, Foxcroft GR, Dyck MK. Seminal plasma proteins as potential markers of relative fertility in boars. J Androl. 2010;31:188-200.

[54] Barranco I, Tvarijonaviciute A, Perez- Patiño C, Alkmin DV, Ceron JJ, et al. The activity of Paraoxonase type 1 (PON-1) in boar seminal plasma and its relationship with sperm quality, function- ality and in vivo fertility. Andrology 2015;3:315-320.

[55] May N, Patterson JL, Pinilla JC, Carpenter A, Werner T, Triemert E, et al. Seminal plasma proteins associated with boar fertility. Reprod Dom Anim 2015;50:110.

[56] Okazaki T, Akiyoshi T, Kan M, Mori M, Teshima H, Shimada M. Artificial insemination with seminal plasma improves the reproductive performance of frozen-thawed boar epididymal spermatozoa. J Androl 2012;33:990-8.

[57] Kirkwood RN, Vadnais ML, Abad M. Practical application of seminal plasma. Theriogenology 2008;70:1364-7.

[58] Robertson SA, Prins JR, Sharkey DJ, Moldenhauer LM. Seminal fluid and the generation of regulatory T cells for embryo implantation. Am J Reprod Immunol. 2013;69:315-30.

[59] Barkalina N, Jones C, Coward K. Nanomedicine and mammalian sperm: Lessons from the porcine model. Theriogenology 2015 doi:10.1016/j.theriogenology.2015.05.025. [Epub ahead of print]. 